Maria Cristina Strausz ${ }^{2}$

Jorge Mesquita Huet Machado ${ }^{2}$

Leila de Souza Rocha Brickus ${ }^{3}$

\section{Análise de um acidente por contaminação fúngica em uma biblioteca pública no município do Rio de Janeiro $^{1}$}

\author{
Analysis of a fungal contamination accident at a public library \\ in Rio de Janeiro
}

${ }^{1}$ Artigo baseado na dissertação de mestrado de Maria Cristina Strausz intitulada Análise de um acidente fúngico em biblioteca: um caso de síndrome do edifício doente, apresentada à Escola Nacional de Saúde Pública, Fiocruz, Rio de Janeiro. 2001. Apresentado no I Seminário Nacional de Saúde e Ambiente no Processo de Desenvolvimento, Rio de Janeiro, 2000, sob o título Análise de um acidente fúngico em biblioteca: um caso de síndrome do edifício doente; e no V Congresso Brasileiro de Epidemiologia, Curitiba, 2002, sob o título Contribuição de ferramentas epidemiológicas na análise de um acidente de contaminação fúngica em biblioteca.

${ }^{2}$ Coordenação de Saúde do TrabaIhador da Fundação Oswaldo Cruz. Rio de Janeiro. Brasil.

${ }^{3}$ Escola Nacional de Saúde Pública Sérgio Arouca da Fundação Oswaldo Cruz. Rio de Janeiro. Brasil.

\begin{abstract}
Resumo
Partindo de um acidente de contaminação por fungos ocorrido em dezembro de 1997 em uma biblioteca pública no município do Rio de Janeiro, foi testada a aplicabilidade de uma proposta metodológica de análise sociotécnica de acidentes, que foi desenvolvida pelo Centro de Estudo de Saúde do Trabalhador e Ecologia Humana (CESTEH/Fiocruz), para análise de acidentes industriais ampliados. $\mathrm{O}$ acidente foi conseqüência de um fato anunciado meses antes, quando os trabalhadores da biblioteca denunciaram, através de um abaixo-assinado, os problemas de descontrole da temperatura ambiente, que se traduzia em desconforto, sintomas respiratórios e afastamento do trabalho, decorrentes daquelas condições. O evento foi caracterizado como um caso de Síndrome do Edifício Doente, que é um problema decorrente da má qualidade do ar de interiores, especialmente em ambientes climatizados artificialmente. Nesse sentido, despertou a atenção dos profissionais de bibliotecas do Brasil para um problema bastante comum, embora pouco estudado, especialmente no campo da saúde pública e, dentro dela, o campo da saúde do trabalhador.
\end{abstract}

Palavras-chaves: análise de acidentes, Síndrome do Edifício Doente, saúde do trabalhador.

\begin{abstract}
The applicability of a methodology to analyze large industrial accidents using social-technical analysis developed by the Center of Studies on Worker's Health and Human Ecology (CESTEH/Fiocruz) was tested in a fungal contamination accident occurred in December 1997 at a public library in Rio de Janeiro. The accident was due to problems in controlling the ambient temperature, which resulted in discomfort, breathing symptoms, and sick leave. The library workers, through a signed petition, had already brought the problem forward. It was characterized as a case of Sick Building Syndrome, which is caused by bad indoor air quality, especially in acclimatized environments. The event made Brazilian librarians aware of an issue which, in spite of being rather ordinary, is still not very well known within the field of Public Health, particularly in the area of Worker's Health.
\end{abstract}

Keywords: accident analysis, Sick Building Syndrome, worker's health. 


\section{Introdução}

Historicamente, os acidentes de trabalho têm sido vistos como eventos de responsabilidade dos trabalhadores envolvidos. A cultura de culpabilização da vítima perpetua-se até os dias de hoje e a Saúde do Trabalhador, enquanto campo interdisciplinar, vem trazendo um novo enfoque sobre os acidentes de trabalho e as doenças dele decorrentes. As Ciências Sociais trouxeram como contribuição uma abordagem sociotécnica dos acidentes de trabalho, enquanto a Ergonomia moderna trouxe uma nova visão do posto de trabalho.

A indústria vem sendo privilegiada como campo de investigação eleito para o desenvolvimento de metodologias de análise dos acidentes de trabalho. Afinal, os acidentes nela ocorridos são conseqüências de necessidades trazidas pela revolução industrial, que transformou o mundo trazendo não só o desenvolvimento, como novos padrões de doenças e acidentabilidade. Mas há outras razões: acidentes industriais adquirem importância epidemiológica por sua gravidade e abrangência. Além de sua complexidade e sua importância econômica, o setor conta com a demanda dos trabalhadores organizados. Dentre outras áreas onde se desenvolvem estas metodologias estão a aeroespacial, de transportes em geral e de energia nuclear, com influência direta sobre as metodologias de análise de acidentes em geral.

A mudança do perfil da atividade econômica no decorrer dos últimos anos nos

\section{$O$ acidente}

Durante as festas de Ano Novo de 1996/7, uma biblioteca pública localizada no município do Rio de Janeiro foi alvo de intensa proliferação de fungos. O evento havia sido precedido por outros problemas, como as constantes queixas relativas à saúde dos funcionários, o que, por sua vez, já tinha justificado a criação de uma comissão de saúde.

Tal comissão tinha como objetivos monitorar a temperatura ambiental e mobilizar os trabalhadores para que se tomassem providências com relação a seu provável desajuste. $\mathrm{O}$ monitoramento revelou que a temperatura encontrava-se muito abaixo do nível de conforto térmico, chegando a $14^{\circ} \mathrm{C}$ no decorrer do dia, um indicativo seguro trouxe a necessidade de desenvolver metodologias de análise de acidentes em outros setores da economia, com ênfase no setor de serviços. Contudo, a transposição das metodologias do setor industrial para outros setores freqüentemente esbarra nas limitações inerentes a todo tipo de adaptação.

O crescimento do setor de serviços, aliado à flexibilização dos contratos e à precarização das relações de trabalho, tem trazido um incremento de exposições a novos riscos ocupacionais e doenças relacionadas ao trabalho. Os Distúrbios Osteoarticulares Relacionados ao Trabalho (DORT) ou as Lesões por Esforços Repetitivos (LER) e a Síndrome do Edifício Doente (SED) são alguns exemplos de síndromes modernas relacionadas diretamente à organização e ao ambiente de trabalho. As LER/DORT, mundialmente estudadas, já se configuram como epidemia. A SED ainda carece de estudos mais profundos em climas tropicais, como no Brasil, embora já seja estudada há mais de vinte anos nos países dependentes de climatização artificial (MOLHAVE, 1992; SKOV, 1992).

Nesse contexto, este estudo analisa um caso de Síndrome do Edifício Doente utilizando como base metodológica uma proposta de análise de acidentes desenvolvida pelo Centro de Estudo de Saúde do Trabalhador e Ecologia Humana (CESTEH) da Fundação Oswaldo Crua (Fiocruz).

de que as condições ambientais estavam influindo negativamente sobre a saúde dos trabalhadores (BARCELLOS et al., 1996).

Strausz (2001) relata que as baixas temperaturas tinham como justificativa a preservação do acervo. No entanto, o sistema de refrigeração era costumeiramente desligado durante a noite, fins de semana e feriados. Deste modo, o aumento da temperatura ambiental, provocado pelo fechamento da biblioteca e o desligamento do seu sistema de refrigeração por vários dias consecutivos, em pleno verão, por ocasião do feriado prolongado do fim do ano de 1996, conjugado ao alto grau de umidade relativa do ar decorrente da intensa chuva no período, forneceram as condições pro- 
pícias para que os fungos, já presentes no ambiente, proliferassem-se intensamente, contaminando todo o ambiente.

Assim, o acidente aconteceu como um fato anunciado meses antes, quando os funcionários denunciaram, através de um abaixo-assinado, as péssimas condições de trabalho, traduzidas em desconforto, dores musculares, de garganta e de ouvido, alergias respiratórias, sinusite, gripe e pneumonia.

$\mathrm{O}$ acidente desencadeou ações de vigilância em saúde do trabalhador na instituição, com o envolvimento de diversos atores sociais, como presidência da instituição, direção da biblioteca, sindicato de trabalhadores, comissão interna de saúde e setores ligados à saúde do trabalhador,

\section{Metodologia}

Segundo Strausz (2001), foi utilizado para a análise do acidente o referencial metodológico da Análise Interdisciplinar e Participativa de Acidentes (AIPA). Desta forma, pode-se identificar e discutir os componentes estruturais disciplinares e seus fatores causais, segundo os seus vários estratos: macroestrutural, no nível da política de administração pública; local, onde se analisa os componentes gerencial, organizacional e tecnológico; e o estrato epidemiológico, onde se revela o impacto do acidente sobre o ambiente e sobre a saúde dos trabalhadores.

A AIPA é uma proposta metodológica criada pelo Centro de Estudos de Saúde do Trabalhador e Ecologia Humana baseada na análise sociotécnica de acidentes industriais desenvolvida por Paté-Cornell, na França, como uma tentativa de superação das atuais abordagens de análise de acidentes industriais, buscando vincular os eventos aos aspectos sociais e gerenciais ou organizacionais na geração dos mesmos (FREITAS \& PORTO, 1997; FREITAS, PORTO \& MACHADO, 2000).

Essa proposta metodológica foi desenvolvida para análise de acidentes químicos ampliados, ou seja, acidentes de alta complexidade. Porém, utilizamo-na em outro campo de estudo: a análise de um acidente em biblioteca, no setor de serviço. A nossa hipótese é de que, no caso do acidente em questão, a AIPA é aplicável devido ao nível de complexidade envolvido nesse caso: além do apoio das unidades técnico-científicas. O diagnóstico inicial indicou que o acidente foi provocado por problemas ligados à climatização da biblioteca.

Este evento foi caracterizado como acidente pelo fato de ter sido um fenômeno agudo de contaminação ambiental, com risco potencial à saúde dos trabalhadores.

As ações desenvolvidas a partir de então visaram preservar a saúde dos trabalhadores, recuperar o acervo e restabelecer condições ambientais que fossem satisfatórias para o conforto dos trabalhadores e dos usuários.

Os problemas relativos ao funcionamento do sistema de ar-condicionado foram muitos e se arrastaram ao longo dos anos subseqüentes. um acidente de contaminação ambiental provocado por fatores gerenciais e tecnológicos, envolvendo as relações sociais do trabalho, com reflexo sobre a saúde dos trabalhadores.

Strausz (2001) informa que foi utilizado pelo órgão responsável pela saúde do trabalhador um inquérito epidemiológico para detecção de queixas relacionadas à exposição que serviu como base para encaminhamentos ao serviço médico. A população em estudo foi dividida em dois grupos, de acordo com a localização do setor de trabalho no prédio: mais expostos e menos expostos. O grupo de trabalhadores mais expostos foi composto de pessoas que desenvolviam suas funções diretamente no acervo, como bibliotecários, estagiários e higienizadores do acervo, ou que circulavam por estes ambientes desenvolvendo as atividades de apoio, como recepção de usuários, limpezas prediais e vigilância. O grupo de trabalhadores menos expostos foi composto de funcionários da área de administração e de informática. Os trabalhadores destes setores foram considerados como menos expostos por não terem contato direto e permanente com o acervo, apesar do sistema de refrigeração propiciar a troca de ar entre os diversos ambientes do prédio, sem distinção. O período em estudo foi de janeiro a setembro de 1997.

Foram colhidas amostras do ar ambiente por uma pesquisadora da instituição, com a finalidade de identificar os 
microrganismos e compostos químicos presentes, e contratada uma assessoria de controle microbiológico para identificar e quantificar os fungos do ambiente, assim como fornecer orientações quanto à sua patogenicidade e aos métodos de higienização do acervo.

O estudo do acidente se deu a partir da análise dos seguintes relatórios: do serviço de saúde do trabalhador; da avaliação ambiental realizada dentro da instituição; de

\section{Resultados da análise}

A contaminação fúngica que determinou o acidente vinha tomando espaço no decorrer de 1996 por problemas no sistema de ar-condicionado. Segundo relatos de funcionários, desde setembro daquele ano, algumas coleções começaram a apresentar focos de contaminação, havendo necessidade de intensificar a rotina de higienização do acervo, apesar das limitações impostas pela carência de pessoal para a execução desta tarefa (BARCELLOS et al., 1996; STRAUSZ, 2001).

É importante ressaltar que, no processo de trabalho em bibliotecas, há um risco biológico controlado devido à presença de fungos e ácaros, principalmente, que têm o papel, o couro, o tecido e a madeira como seu habitat. Este risco pode ser maior ou menor, dependendo de medidas de controle ambiental, como a higienização periódica dos volumes e do ambiente em geral e monitoramento da temperatura e da umidade relativa do ar (VALENTÍN et al., 1998; GAMBALE et al., 1989; CROCE, 1989).

O edifício que abriga a biblioteca, embora tenha sido concebido com a finalidade de acolher o acervo bibliográfico da instituição, teve seu projeto inicial alterado para se tornar um centro de informação científica, com necessidades diferenciadas de climatização (SICT, 1991).

A AIPA se baseia fortemente na análise sociotécnica dos acidentes, em que o componente social se refere à vulnerabilidade social do contexto e dos indivíduos envolvidos, à organização sindical, ao nível de democratização ou hierarquização das relações de trabalho, às políticas de gerenciamento de riscos e, mais globalmente, às políticas sociais e econômicas implementadas no país ou região (PORTO, 1994). uma empresa de controle microbiológico contratada; e da comissão de funcionários constituída meses antes do acidente, além de pareceres técnicos ligados às áreas de engenharia, micologia médica, pneumologia e dermatologia ocupacional e, finalmente, da avaliação clínica e laboratorial dos trabalhadores e de entrevistas com alguns atores institucionais que vivenciaram os momentos pré e pós-acidente.

A instituição pública em questão busca estruturalmente a democratização das relações de trabalho através da eleição interna de seus dirigentes e gestores, além de contar com um sindicato de trabalhadores engajado na luta por melhores condições de trabalho. Eventualmente, a organização dos trabalhadores se dá de forma descentralizada através de comissões de saúde formadas livremente, que fazem interlocução com o órgão de saúde do trabalhador.

Segundo Strausz (2001), no período que antecedeu ao acidente, foi criada uma Comissão de Saúde do Trabalhador na biblioteca a fim de negociar junto à direção alguma solução para o problema de baixas temperaturas no decorrer do período de trabalho. A comissão solicitou a compra de equipamentos para o monitoramento de temperatura e umidade relativa do ar nos diversos ambientes do prédio e passou a recomendar que os trabalhadores se recusassem a trabalhar em temperaturas abaixo de $20^{\circ} \mathrm{C}$. A alternativa à recusa foi a adoção de estratégias, como a abertura de janelas, vedação das saídas de ar-condicionado, até mesmo o desligamento do sistema de refrigeração do prédio.

Ao se deparar com a suspeita de crescimento de fungos nas estantes do acervo e em seus volumes, a comissão colheu amostras e enviou para análise. O resultado confirmou a presença de fungos e fez com que a comissão solicitasse à direção um incremento no contingente de trabalhadores para a higienização do acervo, o que não foi possível devido às limitações para contratação de pessoal na instituição. A comissão levou o problema ao órgão de saúde do trabalhador, solicitando sua mediação junto aos setores de engenharia e arquitetura da instituição (BARCELLOS et al., 1996). 
A vertente tecnológica da AIPA entende o acidente como conseqüência:

de uma ou mais disfunções do processo, em que a característica patológica do sistema tende a se manifestar de forma imediata e abrupta, em oposição às situações "normais" de poluição crônica (...) a isso somadas as contínuas exposições às cargas de trabalho (...) podendo vir a gerar no futuro, doenças graves. (FREITAS et al., 2000, p. 55)

Como disfunção no processo, identificamos o descontrole da temperatura como um fator mais evidente que encobria altas taxas de umidade relativa do ar, trazendo um incremento no desconforto térmico.

Ao contrário da oscilação da temperatura, a umidade relativa do ar permanecia constantemente alta, em torno de $90 \%$ a $100 \%$, o que facilitava a proliferação de fungos no seu habitat: o papel. As altas taxas de umidade no ambiente interno tinham diversas causas, que foram eliminadas uma a uma nos meses que sucederam ao acidente, sendo que grande parte delas estava associada a erros no projeto ou em sua execução (CONTROLBIO, 1998; STRAUSZ, 2001):

- ausência de calhas de escoamento junto ao telhado, o que fazia com que a água da chuva escorresse pelas paredes externas;

- as janelas do prédio não eram totalmente vedadas, o que possibilitava a entrada da umidade do meio externo;

- as janelas de tomada de ar do exterior foram instaladas muito baixas, captando, também, toda a umidade proveniente da evaporação do solo.

O prédio fora construído sobre solo pantanoso, que não foi impermeabilizado em torno do prédio e, portanto, o ar não deveria ser captado nesta altura. O projeto do arcondicionado provavelmente se baseou em tabelas padronizadas de climatização, não levando em conta a localização do prédio:

- má localização da central de água gelada ao lado do prédio, propiciando que a umidade gerada seja levada com o vento ao seu encontro;

- a existência de um jardim interno que se comunicava diretamente com o interior do edifício, cuja base não estava isolada do solo. O projeto original do sistema de arcondicionado permitia a comunicação do ar entre os ambientes. Desta forma, a umi- dade vinda do exterior se distribuía indiscriminadamente junto com o ar frio.

Segundo Strausz (2001), aliados aos problemas no projeto, estavam alguns fatores gerenciais, que podem ser descritos de forma hierarquizada:

- no mecanismo de controle da temperatura, havia válvulas elétricas que estavam queimadas, tornando o ambiente interno extremamente frio no decorrer do ano. $\mathrm{O}$ contrato de manutenção não previa a compra de peças e componentes, o que ficava a cargo do setor de manutenção da instituição e dependente de um lento processo licitatório de compras.

- operação inadequada do sistema de refrigeração. O desligamento do sistema de refrigeração ao final do dia e nos finais de semana provocava choque térmico e condensação dentro dos armazéns. Desde a sua implantação, não foram estabelecidas normas e procedimentos de regulação do sistema de ar-condicionado. A operação do sistema era feita por um funcionário da área administrativa sem conhecimento técnico, pois, naquela ocasião, a Portaria $n^{\circ}$ 3.523/ GM de 1998 (BRASIL, 1998), que recomenda a presença de um técnico para a operação do sistema de ar-condicionado, ainda não estava em vigor. Além do mais, como o ambiente atingia temperaturas muito baixas a partir de metade do expediente, havia muita pressão interna por parte dos funcionários para que o sistema fosse desligado.

A AIPA defende a mudança de foco de análise, com uma abordagem que considera a organização do trabalho e seu papel no evento. Esta mudança implica em ampliar o foco de análise para todo o processo de trabalho, incluindo aí os aspectos gerenciais e organizacionais, deslocando as investigações de como aconteceu para porque aconteceu. Dentro desse contexto, inclui-se a participação dos trabalhadores não mais como geradores, mas como atores no processo de análise do acidente. Daí a denominação "participativa”. No acidente em questão, vemos os fatores ambientais interferindo negativamente no processo de trabalho. A participação dos trabalhadores em todas as etapas, quer seja em discussões de grupos organizados ou como comissão de saúde e associação de funcionários, induzindo uma resposta institucional, foi decisiva para a compreensão do acidente.

A introdução de uma abordagem de análise interdisciplinar enriquece a compreensão dos acidentes, abrangendo uma 
gama de fatores que buscam sistematizar uma hierarquia condicionante do evento. A incorporação das dimensões social, política e ideológica permite ver o trabalhador e seu corpo historicamente determinado e implica na articulação com saberes anteriores, reconstruídos sob uma ótica social (FREITAS, PORTO \& MACHADO, 2000).

\section{Machado (1997, p. 37) afirma que a:}

intervenção interdisciplinar em vigilância tem um potencial de superação das restrições técnicas das abordagens disciplinares. Os componentes tecnológicos, epidemiológicos e sociais dessa ação interdisciplinar atuam como mediadores da relação entre processo de trabalho e saúde. Logo, ao levarmos em consideração esses três componentes estruturais e a dinâmica dessa relação, somos obrigados a pensar e agir interdisciplinarmente.

$\mathrm{O}$ impacto dos fatores relacionados à organização do trabalho aliado aos fatores tecnológicos é percebido sobre o corpo e a mente dos trabalhadores, manifestando-se como sintomas de doenças já conhecidas ou não, intoxicações agudas e crônicas, todos eles dificilmente reconhecidos institucionalmente como acidente de trabalho.

Segundo Freitas, Porto e Machado (2000, p. 59):

A geração histórica dos acidentes configura uma relação hierárquica entre os elementos interdisciplinares múltiplos, dependendo dos setores, empresas e tecnologias analisados, que reorganizam a estrutura de relações entre os elementos sociais, tecnológicos e de saúde.

O componente epidemiológico do acidente em questão é dado pelas avaliações de saúde e ambiental executadas.

A avaliação de saúde foi realizada a partir do inquérito epidemiológico utilizado como ponto de partida para o levantamento de queixas para posterior encaminhamentos a médicos generalistas e especialistas, à avaliação laboratorial e a outros exames complementares.

Segundo Strausz (2001), dentre os 97 trabalhadores examinados, 68 (71,1\%) foram considerados saudáveis ao exame clínico e 14 (14,4\%) com outras alterações não relacionadas à exposição. Apenas 6 pessoas $(6,2 \%)$ apresentaram alterações possivelmente relacionadas à exposição fúngica (manifestações alérgicas respiratórias ou dermatológicas). Cabe ressaltar ainda que 2 trabalhadores mantiveram-se em licença médica por mais de 30 dias e 1 solicitou transferência de unidade.

Foi calculado o risco atribuível (RA) de queixas dermatológicas entre os grupos de diferentes graus de exposição, sendo este de $25 \%$. O mesmo indicador de relação entre exposição e efeito para as queixas respiratórias apresentou um resultado relativamente de menor significância, ou seja, um RA de 5\%, representando um número menor de casos de doenças respiratórias que podem ser atribuídos ao diferencial de exposição decorrente do acidente.

A contagem de eosinófilos no sangue foi destacada por ser um indicador de reação alérgica muito sensível, porém inespecífico. A Tabela 1 revela uma agregação dos casos de eosinofilia com a presença de queixas respiratórias, o que pode estar associado a quadros de alergia respiratória em decorrência da exposição fúngica (MACHADO, STRAUSZ E TOSCHI, 1997; STRAUSZ, 2001).

A eosinofilia foi observada em 18 $(18,9 \%)$ dos 97 trabalhadores avaliados, dos quais 4 apresentaram exame parasitológico de fezes positivo, o que também contribui para o aumento dos eosinófilos. Destes, apenas 1 permaneceu com a alteração na contagem de eosinófilos após o tratamento de parasitose, podendo-se concluir que $15(15,8 \%)$ trabalhadores sofreram algum processo alérgico na ocasião do acidente. Registramos que a grande maioria dos trabalhadores que apresentaram eosinofilia no primeiro exame negativaram após afastamento das atividades e das medidas de aeração do ambiente (BLUMENTHAL, 1984a e 1984b).

Foi também realizada a avaliação imunológica específica para aspergillus, apresentando $100 \%$ de negatividade. Essa avaliação é fundamental devido ao potencial patogênico do aspergillus e sua negativação é bastante importante no sentido da estimativa da gravidade dos achados clínicos que encontraríamos (PENNINGTON, 1986).

Uma vez constatada a contaminação fúngica, foram tomadas diversas medidas de controle, como a aeração dos ambientes através da abertura das janelas, fumigação para eliminação dos fungos e ações de vigilância ambiental, como monitoramento de umidade e temperatura e análise da qualidade do ar para fungos e contaminantes químicos.

Segundo o relatório de Rocha (1998), foram realizadas três amostragens do ar 
Tabela 1 Freqüência de eosinofilia em trabalhadores de biblioteca pública no município do Rio de Janeiro expostos à contaminação fúngica, segundo relato de queixas respiratórias, no período de janeiro a março de 1997

\begin{tabular}{ccc}
\hline Queixas respiratórias & Eosinofilia & Percentual \\
\hline Sim & 11 & $61,2 \%$ \\
\hline Não & 7 & $38,8 \%$ \\
\hline Total & 18 & $100 \%$ \\
\hline
\end{tabular}

interno e externo da biblioteca de Manguinhos durante o ano de 1997. Todos os valores de umidade relativa do ar encontrados no ambiente interno estavam acima de $60 \%$. Na primeira amostragem, durante a infestação de fungos na biblioteca, foi observado que tanto a umidade relativa do ar elevada como a oscilação da temperatura no interior da biblioteca foram fatores de riscos para o acervo e favoreceram a proliferação de fungos. Os valores de umidade relativa do ar no interior da biblioteca chegavam a ser maiores do que no ar externo. Foi observada uma séria umidificação de papéis nas salas de pesquisa e nos armazéns do acervo (ROCHA, 1998).

Os valores aceitos como mais convenientes à conservação de acervos bibliográficos são 55\% de umidade relativa do ar e temperatura entre 20 e $22^{\circ} \mathrm{C}$ (ABNT, 1980). Mais recentemente, a Agência Nacional de Vigilância Sanitária estabeleceu valores de $40 \%$ a $55 \%$ de umidade relativa do ar e 20 a $23^{\circ} \mathrm{C}$ para ambientes de arte, onde se pode incluir acervo bibliográfico (BRASIL, 2000).

As amostragens de junho/97 e setembro/97, após o conserto do sistema de arcondicionado, mostraram claramente que, mesmo com todas as medidas de engenharia adotadas, o sistema de condicionamento do ar da biblioteca não conseguia manter a temperatura e a umidade relativa do ar adequadas para este tipo de ambiente. Esse problema de refrigeração foi detectado desde a inauguração da biblioteca.

Vários gêneros de fungos foram identificados na biblioteca durante a infestação. Sua distribuição entre os pontos de amostragem foi bastante similar. Dos 10 gêneros identificados, por ocasião da primeira amostragem, os mais comuns foram Aspergillus sp. (96,4-164,3 ufc/m³), Aspergillus niger $\left(67,9-153,6 \mathrm{ufc} / \mathrm{m}^{3}\right)$ e Penicillium sp. $\left(78,6-246,4 \mathrm{ufc} / \mathrm{m}^{3}\right)$. Os componentes restantes tiveram uma contribuição moderada onde foram detectados. Os valores de fungos totais estiveram na faixa de 600,0$960,7 \mathrm{ufc} / \mathrm{m}^{3}$.

Em junho/97, após a higienização do acervo e do prédio, os níveis de fungos totais caíram consideravelmente. O gênero mais comum encontrado foi Cladosporium sp. (61,9-183,3 ufc $\left./ \mathrm{m}^{3}\right)$. Os principais fungos identificados em janeiro/97 tiveram um declínio significante: Aspergillus sp. (11,921,9 ufc/ $/ \mathrm{m}^{3}$ ), Aspergillus niger (não detectado) e Penicillium sp. (14,3-26,2 ufc/m³).

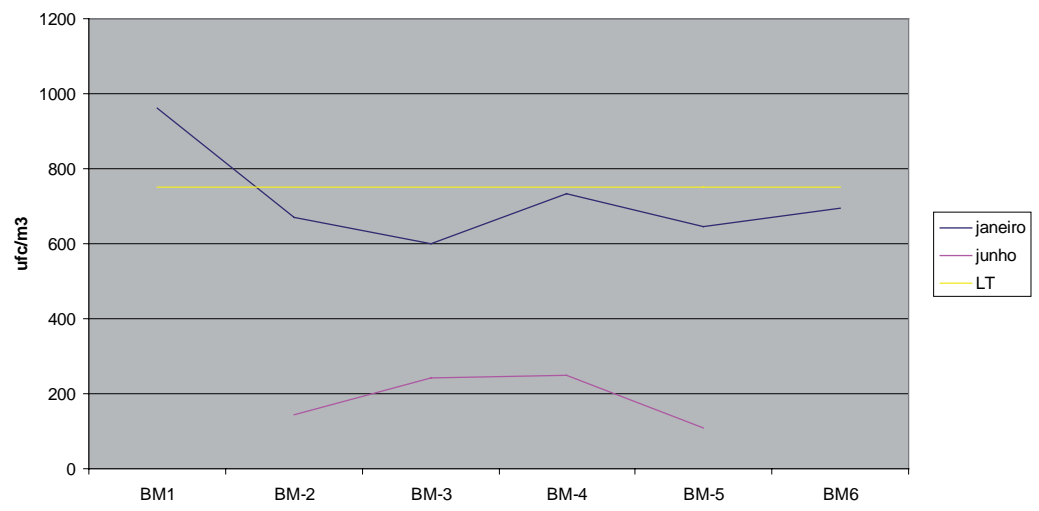

Abreviações: BM-1: Saguão da recepção; BM-2: sala de leitura de periódicos; BM-3: sala de referência; BM-4: armazém A; BM-5: armazém B; e BM-6: sala de informática.

Gráfico 1 Avaliação quantitativa de fungos totais presentes no ar ambiental de biblioteca pública no município do Rio de Janeiro em janeiro e junho de 1997 
Nessa avaliação foi observado que os níveis estavam abaixo do limite máximo aceitável, indicando que a contaminação por fungos estava sob controle. O Gráfico 1 demonstra a distribuição quantitativa de fungos totais em seis pontos de amostragem na biblioteca estudada em janeiro e junho de 1997.

Com relação à avaliação de contaminantes químicos, foram realizadas avaliações dos teores de partículas totais em suspensão (PTS), dos compostos orgânicos voláteis totais (COVT) e dos aldeídos. A determinação do teor de partículas inaláveis em suspensão é de extrema importância para avaliar os possíveis efeitos adversos à saúde humana devido à presença de material particulado. As amostragens foram feitas para partículas totais em suspensão devido ao fato de se possuir ciclones suficientes para realizar a amostragem em vários pontos simultaneamente naquela ocasião (BRICKUS e NETO, 1999).

Normalmente, a maior fonte de PTS no ar de interiores é a atividade humana, assim como a incursão do ar externo. Analisando-se os pontos internos amostrados de PTS, foi observado que o BM-1, referente ao saguão de recepção da biblioteca, apresentou valor máximo, seguido por BM-2, referente à sala de leitura de periódicos, também bastante freqüentada pelos usuários. Esse dado comprova a idéia de que, em recintos fechados, as atividades humanas são uma das fontes principais de PTS. Ao mesmo tempo, reduz a importância da influência poluidora externa sobre o ambiente interno.

Síntese das situações evidenciadas pela análise:

\section{Discussão}

O estudo nos mostra que a AIPA é uma proposta metodológica perfeitamente aplicável a este acidente ao possibilitar elencar os seus fatores condicionantes de forma sistematizada e hierarquizada.

Além disso, podemos inferir sobre a sua aplicação em outros acidentes com o mesmo nível de complexidade, independentemente do setor da economia. A AIPA traz à tona os problemas gerenciais como geradores potenciais de acidentes de trabalho, enquanto as metodologias tradicionais de análise de acidentes apontam o fator humano como seu principal gerador.

\section{Problemas no projeto arquitetônico}

- pouca experiência das empresas brasileiras em construção de bibliotecas e prédios onde há grande número de publicações e a necessidade de conservação de acervo;

- capacidade relativa de instância pública em acompanhar o desenvolvimento de projeto desse porte, principalmente quanto ao item de refrigeração e de exaustão do ambiente;

- o local escolhido é extremamente sujeito a variações intensas de temperatura e umidade, exigindo um mecanismo de controle de alta complexidade.

\section{Problemas gerenciais}

- é priorizado o controle de umidade via redução de temperaturas, desencadeando desconforto para as pessoas;

- há uma demora de resposta institucional na intervenção, embora tenha havido queixas ambientais e mesmo clínicas dos trabalhadores.

\section{Problemas ambientais}

- temperatura e umidade descontroladas;

- proliferação de fungos;

- desconforto térmico generalizado.

\section{Problemas epidemiológicos}

- há uma situação de aumento na incidência de casos de doenças respiratórias leves e alergias respiratórias e de pele. Esse efeito dermatológico é considerado específico para esse tipo de contexto de contaminação fúngica.

A aplicabilidade da AIPA neste acidente também se deu por tratar-se de uma situação de descontrole ambiental, gerando exposições múltiplas a agentes físicos e biológicos, levando a efeitos de complexidade variável.

A infestação fúngica da biblioteca ocorrida em dezembro/96 foi um evento caracterizado como "Síndrome do Edifício Doente" e despertou o interesse de outras bibliotecas do Rio de Janeiro e de outros estados por apontar para um tipo de problema pouco discutido entre os trabalhadores desta atividade. Apesar de haver uma vasta 
bibliografia sobre conservação de acervos e arquitetura de bibliotecas, poucos estudos discutem a saúde desses trabalhadores.

Embora tenha sido observado empiricamente que os trabalhadores que realizam suas atividades em bibliotecas são com freqüência acometidos por problemas de saúde relacionados ao ambiente de trabalho, ainda não há estudos que comprove o que a prática evidencia. Estudar o tema e divulgar os resultados dá visibilidade às questões de saúde desses trabalhadores e ambientes. Dessa forma, a vigilância à saúde dos trabalhadores de bibliotecas passa a

\section{Referências}

ABNT. Associação Brasileira de Normas Técnicas. NBR 6401: Instalações centrais de ar-condicionado para conforto - parâmetros básicos de projeto. Rio de Janeiro: ABNT, 1980.

BARCELLOS, C. et al. Relatório de diagnóstico e propostas de solução para o sistema central de ar-condicionado do prédio da Biblioteca Central de Manguinhos. Rio de Janeiro: [s.n.], 1996. Mimeografado.

BLUMENTHAL, D. S. Ascaridíase. In: WYNGAARDEN, J. B.; SMITH Jr., L. H. Cecil: Tratado de Medicina Interna. 16. ed. Rio de Janeiro: Interamericana, 1984a. p. 1795-1796.

. Estrongiloidíase. In:

WYNGAARDEN, J. B.; SMITH Jr., L. H. Cecil: Tratado de Medicina Interna. 16. ed. Rio de Janeiro: Interamericana, 1984b. p. 1792-1793.

BRASIL. Resolução Anvisa no 176, de 24 de outubro de 2000. Brasília, DF, 2000.

. Portaria $\mathrm{n}^{\mathrm{O}} 3.523$, de 31 de agosto de 1998. Diário Oficial da União, n. 166, Brasília, DF, 1998.

BRICKUS, L. S. R.; NETO, F. R. A. A qualidade do ar de interiores e a química. Rev. Química Nova, Viçosa, v. 22, n. 1, p. 1-9, 1999.

CONTROLBIO. Laudo Técnico. Rio de Janeiro: [s.n.], 1997. Mimeografado.

CROCE, J. Fungos ambientais e alergias respiratórias. In: SEMINÁRIO SOBRE PRESERVAÇÃO DE BENS CULTURAIS. SISTEMA INTEGRADO DE BIBLIOTECAS, 1, 1989, São Paulo. Anais..., ser tema de particular atenção por ser uma população de trabalhadores exposta ocupacionalmente a riscos físicos, químicos e biológicos decorrentes de climatização artificial. Ao contrário da biblioteca em questão, muitas vezes as bibliotecas estão instaladas em áreas adaptadas, em subsolos de edifícios, com problemas crônicos de manutenção.

A publicidade adversa desta infestação fúngica gerou não apenas solidariedade de outras bibliotecas brasileiras como também pedido de ajuda de diferentes partes do país.

São Paulo: Universidade de São Paulo, 1989.

FREITAS, C. M.; PORTO, M. F. S. Aspectos sociais e qualitativos nas análises de causas de acidentes industriais em sistemas tecnológicos complexos. Revista Produção, São Paulo, v. 7, n. 1, p. 33-55, 1997.

FREITAS, C. M; PORTO, M. F. S.; MACHADO, J. M. H. (Org.). Perspectivas para uma análise interdisciplinar e participativa de acidentes (AIPA) no contexto da indústria de processo. In:__. Acidentes industriais ampliados: desafios e perspectivas para o controle e a prevenção. Rio de Janeiro: Fiocruz, 2000. cap. 1.

GAMBALE, W. et al. Fungos do ambiente em bibliotecas da Universidade de São Paulo e relação com alergias respiratórias. In: SEMINÁRIO SOBRE PRESERVAÇÃO DE BENS CULTURAIS. SISTEMA INTEGRADO DE BIBLIOTECAS, 1, 1989, São Paulo. Anais... São Paulo: Universidade de São Paulo, 1989. p. 27-37.

MACHADO, J. M. H. Processo de vigilância em saúde do trabalhador. Cad. Saúde Pública, Rio de Janeiro, v. 13, supl. 2, p. 33-45, 1997.

MACHADO, J. M. H.; STRAUSZ, M. C.; TOSCHI, W. D. Proteção à saúde do trabalhador: informe sobre o acidente de contaminação fúngica na biblioteca de Manguinhos-Fiocruz. Revista Brasindoor, São Paulo, v. 7. p. 4-10, 1997.

MOLHAVE, L. Controlled experiments for studies of the silk building syndrome. Ann. N.Y. Acad. Sci., v. 641, p. 697-711, 1992. 
PENNINGTON, J. E. Aspergillus. In: SAROSI, G. A.; DAVIES, F. F. Fungal diseases of the lung. Orlando: Grune \& Stratton, 1986. p. 175-189.

PORTO, M. F. S. Trabalho industrial, saúde e ecologia: avaliação qualitativa de riscos industriais em dois estudos de caso na indústria química. 1994. Tese (Doutorado) - Coordenação dos Programas de PósGraduação em Engenharia, Universidade Federal do Rio de Janeiro, Rio de Janeiro, 1994.

ROCHA, L. S. Relatório final das atividades do projeto biblioteca de Manguinhos e a síndrome do edifício doente. CNPQ, Coordenação do Programa RHAE. Rio de Janeiro: [s.n.], 1998.

SICT. Estudo para implantação da nova biblioteca de Manguinhos. [S.l.: s.n.], 1991. Mimeografado.

SKOV, P. The silk building syndrome. Ann. N.Y. Acad. Sci., v. 641, p.17-20, 1992.

STRAUSZ, M. C. Análise de um acidente fúngico em biblioteca: um caso de síndrome do edifício doente. 2001. Dissertação (Mestrado) - Escola Nacional de Saúde Pública, Rio de Janeiro, 2001

VALENTÍN, N. et al. Microbial control in archives, libraries and museums by ventilation systems. Rev. Restaurator, n. 19, p. 85-107, 1998. 